\title{
PROPUESTA DE PLATAFORMA DE NOTIFICACIONES DURANTE TRATAMIENTOS EN LA SALA DE ONCOLOGÍA DEL HOSPITAL SAN FELIPE
}

ISSN 2219-6722

ISSNE 2222-2707

LUIS MIGUEL LÓPEZ, Departamento de Ingeniería en Sistemas Universidad Nacional Autónoma de Honduras UNAH, Ciudad Universitaria Móvil: 3177 - 4673 / 3355 - 3524

\section{RESUMEN}

En Latinoamérica especialmente en Honduras no contamos con sistemas en diversas áreas críticas, una de esas áreas es la salud pública, puede contarse con personal para esta área pero no se tiene el uso de tecnología que permita que los procesos evolucionen. En Oncología, la cual maneja información que es de vital importancia en la salud de las personas que padecen de algún tipo de cáncer, no sea realizado ningún progreso para la atención de estos pacientes, el objetivo es tener una propuesta de una plataforma de notificaciones durante tratamientos en la sala de oncología, deberá proveer a los pacientes y personal del hospital el control para el seguimiento de todos los pacientes, mediante los dispositivos tecnológicos a los que se tiene acceso hoy en día, este proyecto se desea sea el detonante para el desarrollo de las tecnología en el área de la salud. Se orientó para esta área en específico debido la importancia que tiene el tratamiento puntual en el progreso del paciente.

Palabras clave: TIC, Salud, Oncología, Automatización. 


\title{
PROPOSED PLATFORM FOR NOTIFICATION DURING TREATMENTS IN ONCOLOGY WARD OF THE HOSPITAL SAN FELIPE
}

ISSN 2219-6722

ISSNE 2222-2707

LUIS MIGUEL LÓPEZ, Departamento de Ingeniería en Sistemas Universidad Nacional Autónoma de Honduras UNAH, Ciudad Universitaria Móvil: 3177 - 4673 / 3355 - 3524

\begin{abstract}
In Latin America, especially in Honduras we do not have systems for several critical areas. One of these areas is public health, there is staff on this area but there is no technology that allows processes to evolve. In Oncology, which manages information that is vital to the health of people suffering from any type of cancer, no progress has been made for the care of these patients. The goal is to have a proposal for a platform of notifications during treatments in oncology; it shall provide patients and hospital staff a control to monitor all patients through technological devices that are accessed today. It is hoped that this project could be the trigger for the development of technology in the area of health. It was oriented to this specific area because of the importance of punctuality in the treatment of the patient's progress.
\end{abstract}

Keywords: ICT, Health, Oncology, Automation. 


\section{INTRODUCCIÓN}

En el área de la salud se realiza muchas investigaciones y avances, por el hecho se ser Honduras un país en desarrollo no se tiene el beneficio inmediato de ello. En tecnología los centros médicos tienen muchas deficiencias por la falta de inversión. En Oncología del Hospital San Felipe se han logrado grande mejoras en la atención en tratamientos pero por la afluencia de personas se vuelve más complejo el manejo y control de los historiales, tratamientos y citas de los pacientes, por ello la investigación aborda la necesidad del control mediante una plataforma que interactúe con el personal de la Sala y los pacientes que reciben servicios hospitalario.

Para áreas críticas específicamente el área de Oncología, no cuenta con un progreso a nivel tecnológico que provea de atención medica automatizada, no existe sistema de información tecnificado que provee de estadísticas, evolución y casos clínicos y te facilite la interacción con los pacientes. El hospital San Felipe es uno de los primero en el país, asimismo la sala de oncología es de las primeras y más reconocidas sala en el tratamiento de cáncer a nivel nacional, no obstante carece de un sistema que lleve el seguimiento del paciente en los tratamientos contra el cáncer.

La investigación se desarrolla en el Hospital San Felipe de Tegucigalpa por su longevidad, también la cantidad de personal que labora, la cantidad de pacientes de la sala y los tratamientos que se practica los pacientes. Hoy en día la tecnología es accesible a la mayoría de la población por lo que vuelve viable un proyecto de esta índole, sin embargo debe buscarse cómo llegar a los que no poseen este acceso a tecnologías modernas.

Actual en Honduras una de las áreas que sufren por falta de apoyo al desarrollo tecnológico, es la Salud Publica. Los centros hospitalarios no cuenta con equipos suficientes y los que se posee están desactualizados impidiendo el progreso. Analizando las infraestructuras de redes, no se encuentra diseñas conforme a estándares y en otros casos no existe. Muchas de las ayuda tecnologías externas queda abandonadas por falta de las mismas.

\section{Hipótesis:}

El índice de mortalidad en personas con cáncer se reduce si lleva un seguimiento estricto de procedimientos y tratamientos para el cáncer.

El porcentaje de pacientes o familiares de pacientes que dispone de dispositivos móviles es mucho mayor que el porcentaje de los que no dispone de estos dispositivos. 


\section{BREVE HISTORIA DEL HOSPITAL SAN FELIPE}

Nació en 1861 pero, fue inaugurado el 27 de agosto de 1882; localizado en el edificio de los antiguos Ministerios de Gobernación y Defensa, actualmente Museo de la Identidad. 40 años después se trasladó al asilo de indigentes San Felipe en el sitio denominado Sabana Grande donde se ubica actualmente y con ello se amplían sus unidades, a pesar del desmembramiento de servicios y sus traslados hacia los hospitales Materno Infantil (1969) y Escuela (1978), continua en pie de lucha y modernización de sus antigua estructura. ("Historia del Hospital San Felipe").

La misión del Hospital San Felipe es una institución dedicada a brindar servicios salud de la más alta calidad a las personas que así lo demanden, tratando de contar con los mejores servicios a un precio accesible. Para esto cuenta con personal altamente capacitado y con experiencia, dada nuestra filosofía de atención y satisfacción del paciente. Todo esto permite generar recursos para el adecuado funcionamiento de la institución, proyectándose con la sociedad y cumpliendo con su responsabilidad con el ambiente. ("Historia del Hospital San Felipe"). La visión del Hospital es; convertirnos en los líderes de los Hospitales Públicos, a nivel nacional en la rama de atención al paciente, integrando los valores fundamentales de humanismo, profesionalismo y ética médica con las disciplinas centrales de la medicina contemporánea razonamiento clínico cuantitativo y epidemiología clínica, rehabilitación, control de calidad de la práctica médica, administración de recursos y manejo de la información clínica; todo en un escenario que asegure la continuidad de la atención integral del paciente tanto en el área hospitalaria como ambulatoria. ("Historia del Hospital San Felipe")

A su vez, el objetivo general de la investigación es garantizar la correcta ejecución de la gestión y conducción de los procesos en la provisión de servicios hospitalarios. ("Historia del Hospital San Felipe”). Los objetivos específicos de la institución es Dirigir, coordinar, supervisar, monitorear, evaluar y ejecutar en forma pro-activa y participativa, todos los procesos realizados en el trabajo de equipo en el nivel hospitalario. ("Historia del Hospital San Felipe”). 
TABLA No. 1. Atención Directa a Pacientes externos

\begin{tabular}{|l|r|r|r|}
\hline \multicolumn{1}{|c|}{ AREA } & \multicolumn{1}{|c|}{ DIARIAS } & MENSUALES & \multicolumn{1}{c|}{ ANUALES } \\
\hline Cirugía & 33 & 547 & 6,565 \\
\hline Cirugia menor & 6 & 102 & 1,218 \\
\hline Clinica del Diabético & 101 & 1,701 & 20,414 \\
\hline Dermatología & 21 & 362 & 4,342 \\
\hline Medicina General & 364 & 6,132 & 73,584 \\
\hline Medicina Interna & 51 & 856 & 10,266 \\
\hline Medicina Física y Rehabilitación & 49 & 829 & 9,948 \\
\hline Odontología & 45 & 761 & 9,137 \\
\hline Oncología & 101 & 1,698 & 20,376 \\
\hline Oftalmología & 171 & 2,879 & 34,549 \\
\hline Ortopedia & 13 & 224 & 2,687 \\
\hline Otorrinolaringología & 23 & 395 & 4,743 \\
\hline Pediatria & 165 & 2,775 & 33,297 \\
\hline Psicología adulto & 4 & 61 & 733 \\
\hline Psicologia infantil & 8 & 138 & 1,651 \\
\hline Psiquiatria & 27 & 452 & 5,425 \\
\hline Reumatología & 27 & 460 & 5,523 \\
\hline Urología & 12 & 209 & 2,502 \\
\hline Partos & 36 & 612 & 7,347 \\
\hline Cesarea & 6 & 101 & 1,214 \\
\hline TOTAL & $\mathbf{1 , 2 6 5}$ & $\mathbf{2 1 , 2 9 3}$ & $\mathbf{2 5 5 , 5 2 1}$ \\
\hline
\end{tabular}

Fuente: Construcción propia

TABLA No. 2. Atención de servicios de apoyo a Pacientes externos

\begin{tabular}{|l|r|r|r|}
\hline \multicolumn{1}{|c|}{ AREA } & \multicolumn{1}{|c|}{ DIARIAS } & MENSUALES & \multicolumn{1}{c|}{ ANUALES } \\
\hline Banco de Sangre & 0 & 1 & 17 \\
\hline Centro Quirúrgico & 16 & 271 & 3,252 \\
\hline Cocina & 193 & 3,249 & 38,982 \\
\hline Electrocardiograma & 23 & 386 & 4,633 \\
\hline Electromiografia & 3 & 44 & 525 \\
\hline Farmacia & 1,451 & 24,432 & 293,186 \\
\hline Fisioterapia & 3,812 & 64,168 & 770,021 \\
\hline Laboratorio Clinico & 1,539 & 25,904 & 310,846 \\
\hline Mastografia & 1 & 14 & 171 \\
\hline Órtesis y Prótesis & 5 & 79 & 949 \\
\hline Patologia - Biopsias & 5 & 81 & 972 \\
\hline Quimioterapia & 27 & 454 & 5,445 \\
\hline Radiologia & 32 & 540 & 6,475 \\
\hline Radioterapia & 45 & 753 & 9,036 \\
\hline Terapia ocupacional & 515 & 8,675 & 104,099 \\
\hline Trabajo Social & 37 & 625 & 7,498 \\
\hline Ultrasonidos & 20 & 332 & 3,988 \\
\hline TOTAL & $\mathbf{7 , 7 2 3}$ & $\mathbf{1 3 0 , 0 0 8}$ & $\mathbf{1 , 5 6 0 , 0 9 5}$ \\
\hline
\end{tabular}

Fuente: Construcción propia 
TABLA No. 3. Atención Directa a Pacientes internos

\begin{tabular}{|l|r|r|r|}
\hline \multicolumn{1}{|c|}{ ÁREA } & No DE CAMAS & PACIENTES & Dóas \\
\hline $\begin{array}{l}\text { Cirugía / Ortopedia / Cir. Menor / } \\
\text { ORL / Urología }\end{array}$ & & & \\
\hline Asilo de Inválidos & 41 & 1.713 & 8.436 \\
\hline Medicina Interna & 121 & 680 & 20.468 \\
\hline Oncología & 40 & 1.097 & 9.630 \\
\hline Oftalmología & 65 & 2.125 & 18.570 \\
\hline Pediatría & 36 & 739 & 5.641 \\
\hline TOTAL & 30 & 1.091 & 4.937 \\
\hline
\end{tabular}

Fuente: Construcción propia

TABLA No. 4. Atención de servicios a Pacientes internos

\begin{tabular}{|l|r|r|r|}
\hline \multicolumn{1}{|c|}{ AREA } & DIARIAS & MENSUALES & ANUALES \\
\hline Banco de Sangre & 11 & 187 & 2,244 \\
\hline Centro Quirúrgico & 17 & 283 & 3,394 \\
\hline Cocina & 480 & 8,082 & 96,988 \\
\hline Electrocardiograma & - & 62 & 748 \\
\hline Electromiografia & 237 & - & 47,973 \\
\hline Farmacia & 302 & 3,998 & 61,103 \\
\hline Fisioterapia & 378 & 5,092 & 76,447 \\
\hline Laboratorio Clínico & - & 6,371 & - \\
\hline Mastografía & - & - & - \\
\hline Órtesis y Prótesis & 10 & - & 1,939 \\
\hline Patología - Biopsias & 6 & 162 & 1,168 \\
\hline Quimioterapia & 11 & 97 & 2,238 \\
\hline Radiología & 2 & 187 & 457 \\
\hline Radioterapia & 38 & 38 & 7,623 \\
\hline Terapia ocupacional & 97 & 635 & 19,658 \\
\hline Trabajo Social & 4 & 1,638 & 793 \\
\hline Ultrasonidos & $\mathbf{1 , 5 9 8}$ & $\mathbf{2 6 , 8 9 8}$ & $\mathbf{3 2 2 , 7 7 3}$ \\
\hline TOTAL & & & \\
\hline
\end{tabular}

Fuente: Construcción propia

\subsection{El cáncer y su Impacto}

Actualmente una de las enfermedades que ha impactado en la población mundial es el cáncer. La Organización Mundial de la Salud (OMS, 2015) publicó "El cáncer es una de las principales causas de morbilidad y mortalidad en todo el mundo; en 2012 hubo unos 14 millones de nuevos casos y 8,2 millones de muertes relacionadas con el cáncer." (World Health Organization), es importante que se logre disminuir las cifras de mortalidad de los pacientes diagnosticados con cáncer.

En Honduras y los países cercanos a tenido aumentos considerable de personas diagnosticas con cáncer. La OMS público que "60\% de los nuevos casos anuales totales del mundo se producen en África, Asia, América Central y Sudamérica. Estas regiones representan el 70\% de las muertes por cáncer en el mundo"(World Health Organization). Estos datos ponen en alerta a 
la población debido a que los pronósticos van en aumento.

El tratamiento para cada paciente es personalizado por ello de llevársele un seguimiento pero con las cantidades de personas que asisten a los centros hospitalarios vuelve complejo su atención. Si se lleva un tratamiento correcto aumenta las posibilidades de erradicar el cáncer, en el caso de persona con etapa avanzada les permite una mejor calidad de vida. ("Tratamiento contra el cáncer," 2015)

Entender los tipos de cuidados a su disposición es de ayuda para que usted y su médico desarrollen un plan de tratamiento personalizado que tenga en cuenta sus necesidades, objetivos y preferencias personales. Obtenga más información sobre la planificación de los cuidados del enfermo con cáncer en etapa avanzada. (World Health Organization)

\subsection{TIC en la Salud}

Hoy en día el uso de la tecnología en diversas áreas es una realidad, es requerido que los profesionales tengan el conocimiento del uso correcto de las tecnologías. En el área de salud las tecnologías de Información y comunicación dan soporte en los procesos, dando soluciones eficientes y eficaces tanto en documentaciones, generación de imágenes, resultados de laboratorio, dando el surgimiento de e-salud, telesalud y telemedicina. ("Importancia de las TIC (tecnologías de la información y la comunicación) en el cuidado de la salud”).

Con el uso de la TIC surgen nuevos conceptos en salud para uso de tecnologías especializadas hacia esta área, "eSalud, la aplicación de las Tecnologías de Información y Comunicación (en adelante, TIC) en todos aquellos aspectos que afecten al cuidado de la salud, incluyendo entre ellas el diagnóstico, el seguimiento de los pacientes y la gestión de las organizaciones implicadas en estas actividades. De esta manera, con la eSalud, los profesionales tienen la oportunidad de mejorar su acceso al historial clínico de los pacientes, prescribir -de manera electrónica- diferentes recetas, así como consultar las principales publicaciones médicas especializadas., A pesar de los avances eSalud en los hospitales y centros de salud a nivel nacional se sigue trabajo si mayor uso de tecnologías. (“¿Qué es la eSalud? Definición y concepto”)

Las TIC son herramientas de acceso a la información que enfocada en el área de la salud permiten salvar vidas, cambiando la perspectiva del entorno de las áreas de los centro de salud, hospitales y clínicas. Los sistemas en tiempo real que permite dar diagnósticos tempranos y precisos, promueve una evolución tanto en el área pública como privada (Digital).

El progreso de la salud en cuanto a tecnología mejorar los tiempos de respuesta, "Gracias al nacimiento y aplicación de la eSalud, los pacientes tienen la oportunidad de acceder a la atención médica con independencia del lugar en el que se encuentren, fomentando de esta manera la prevención, el bienestar y la vida independiente y autónoma de las personas", los pacientes 
permanecen tiempos menos prologados para los tramites que se realizan, es de esta manera que se da una mejor atención por medio de las TIC

Para la implementación de nuevas tecnología es necesario evaluar los procesos "'La necesidad de nuevos modelos de atención para la cronicidad, la importancia de medir realmente la práctica clínica y la actividad sanitaria, y la importancia de la implantación de las TIC como herramienta clave para mejorar la prestación sanitaria “. ("El 70 por ciento de la atención hospitalaria se podría llevar al hogar”), además de establecer la administración de toda la tecnología utilizada para el servicio y su mantenimiento.

\section{METODOLOGÍA}

La investigación científica parte del enfoque Cuantitativo es deductivo, debido al uso de encuestas, recolección de datos, preguntas o hipótesis, patrones y experimentación. La presente propuesta de plataforma requirió conocer las necesidades de los pacientes y el personal del área de Oncología del Hospital San Felipe.

\section{a. Componentes y recolección de datos}

Para la elección del medio por el cual se capturaron los datos necesarios que permitan el desarrollo de esta investigación, debe ser válido y confiable.

La encuesta cerrada resulta el medio que cumple las necesidades que la investigación exige, a pesar de ser cerrada se incluyó una pregunta que busca conocer las necesidades que no se han tomado en cuenta en el planteamiento de la propuesta.

En la determinación de la muestra influye:

- Variabilidad de población diaria que asiste al centro médico.

- Variabilidad de población que recibe tratamientos en la sala Oncológica.

- Variabilidad de tratamientos aplicados a diario.

Lo anterior nos hace establecer una población desconocida, además el hospital no cuenta con estadísticas exactas de la atención en esta sala.

Se estableció un porcentaje de seguridad de $90 \%$ y $10 \%$ de error, con una muestra de 68 personas, la encuesta se realizo a 35 personas, lo que representan el $51.47 \%$ de la muestra y la determino como aceptable para los fines de esta investigación.

Donde $\mathrm{N}$ es el valor de la muestra.

$\mathrm{Z}=1.645$ al cuadrado con un $90 \%$ de seguridad

$\mathrm{E}=\mathrm{El}$ margen de error de un $10 \%$

$\mathrm{P}$ y $\mathrm{Q}=0.50$ 
Por lo que:

$\mathrm{N}=((1.645) \wedge 2)^{\star}(0.5)^{\star}(0.5) /((0.10) \wedge 2)$

$\mathrm{N}=67.65=68$

\section{ANÁLISIS DE DATOS}

Para el análisis de los resultados obtenidos durante la aplicación de la encuentra se buscó consultar tanto a hombres y mujeres, de manera aleatoria se tuvo una muestra representada en la figura \#1.

Figura No. 1. Genero del encuestado

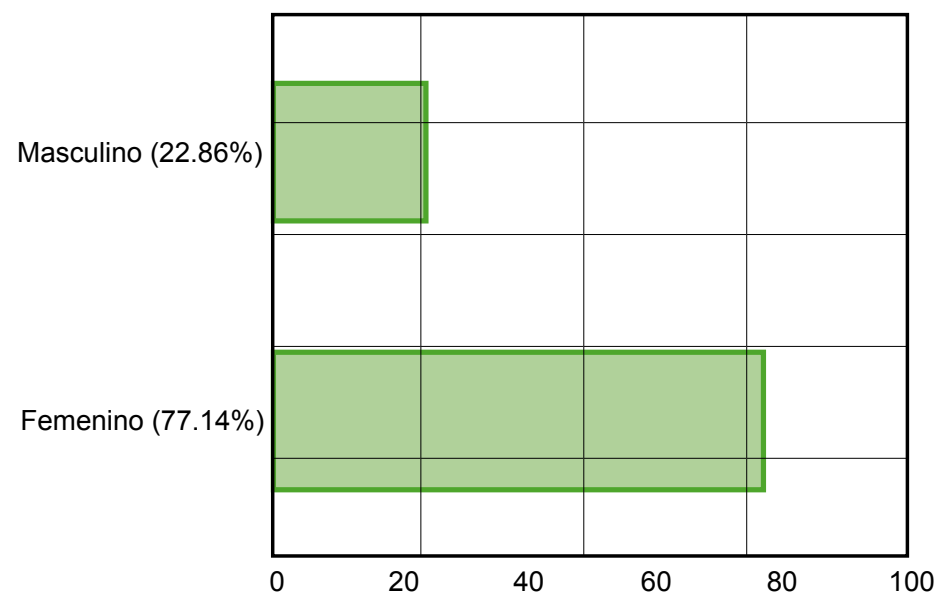

Con este hallazgo establecemos que la mayoría de la población que asiste a este centro hospitalario son mujeres, son más los pacientes femeninos diagnósticos y que recibe tratamiento.

Para la implementación de una planta es necesario conocer los usuarios por eso es importante conocer el rango de edad en el que se encuentra la población y la zona de procedencia (figura \#2, figura \#3).

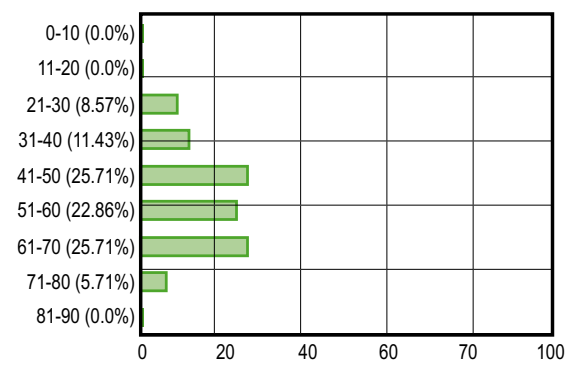

Figura No. 2. Rango de edades

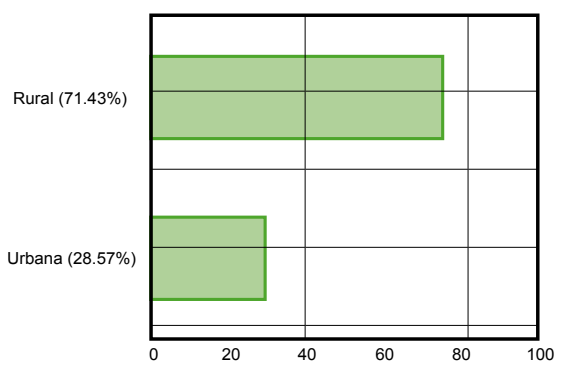

Figura No. 3. Zona de que proviene 
La propuesta tiene como elemento fundamental las notificaciones durante tratamientos, los tratamientos que se aplican deben llevarse con exactitud para erradicar las células cancerígenas, apreciamos en la figura \#4 que el tratamiento más aplicado es la quimioterapia.

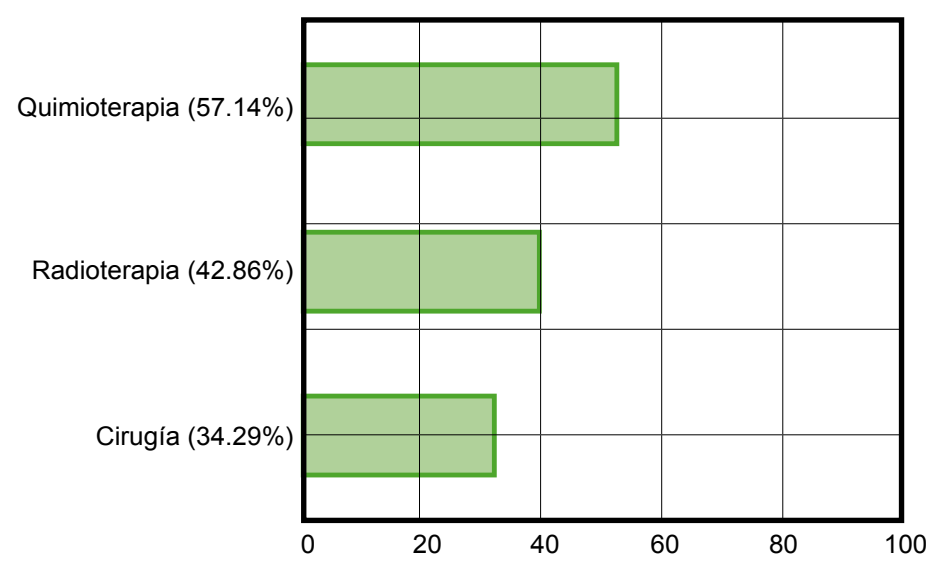

Figura No. 4. Tratamiento Recibido

Uno de los problemas que más expresan los pacientes de la Sala de Oncología, es el manejo de citas y reprogramación por diversas circunstancias (Figura \#5, figura \#6,).

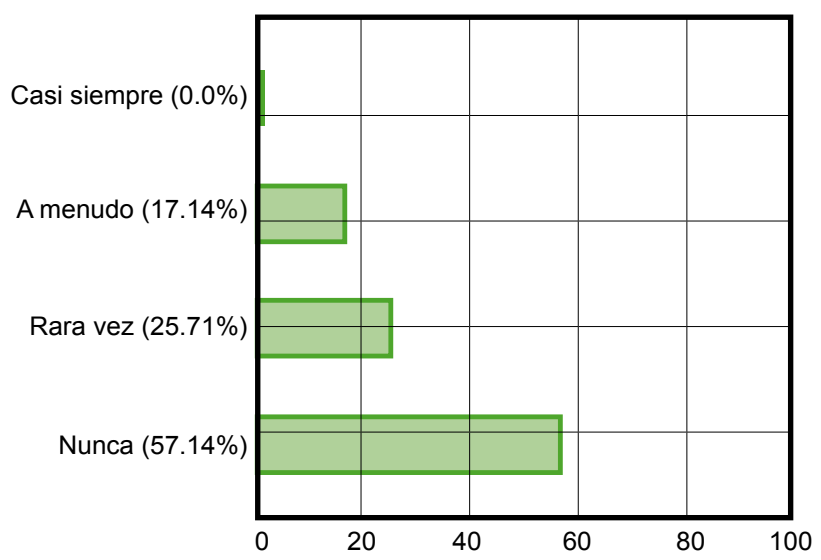

Figura No. 5. Reprogramación de citas para tratamientos 


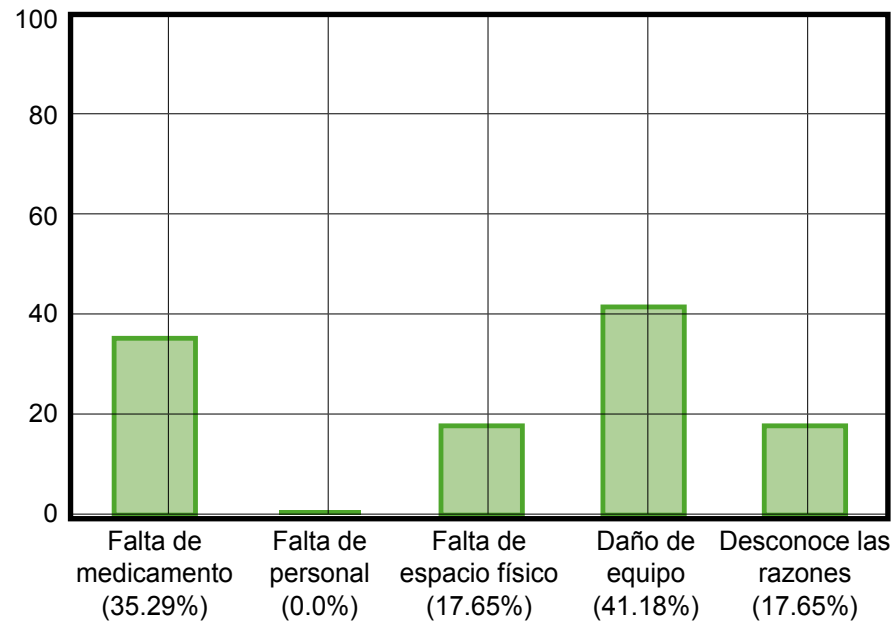

Figura No. 6. Circunstancias que han causado la reprogramación

Para la implementación de una plataforma tecnología que brinde los servicios de seguimiento de tratamientos que se le aplican al paciente, requerimos conocer el acceso a tecnologías, dispositivos de los pacientes o algún pariente cercano (figura \#7, figura \#8).

Si $(97.14 \%)$

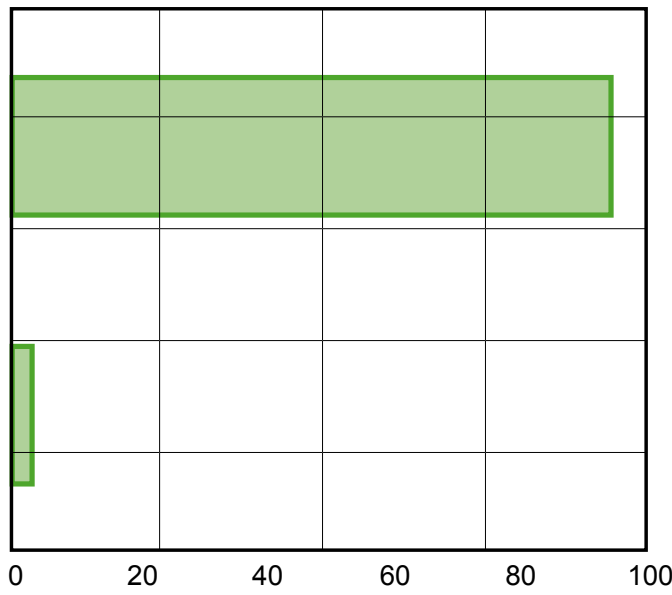

Figura No. 7. Número de personas que cuentan con teléfono celular 


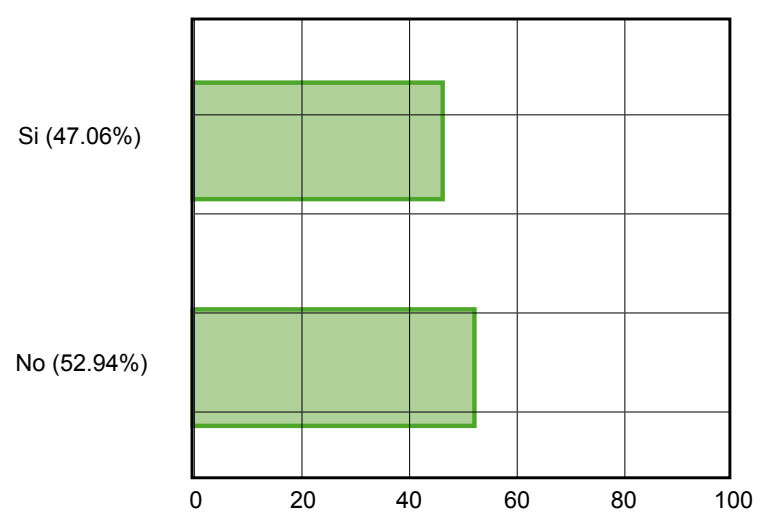

Figura No. 8. Número de personas que cuentan con computadora portátil o de escritorio

También se debe tomar en cuenta las plataformas que posee los pacientes, sistemas operativos, acceso a internet y con qué tipos de servicios cuenta en sus dispositivos (figura \#9, figura \#10, figura \#11, figura \#12).

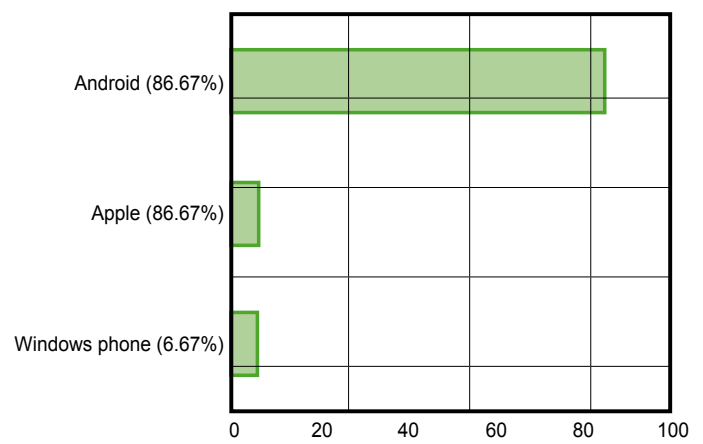

Figura No. 9. Sistema en el dispositivo

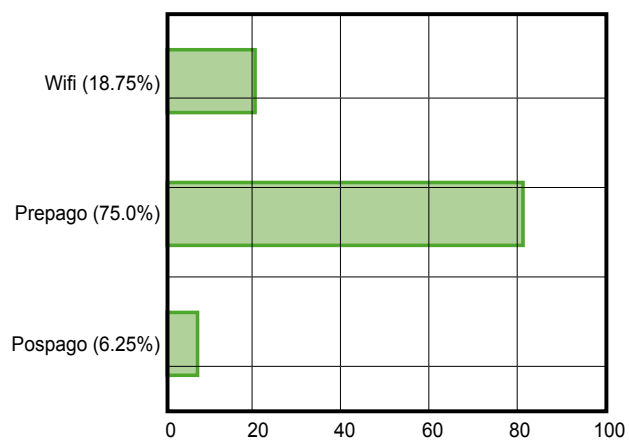

Figura No. 10. Formas de acceder al internet 


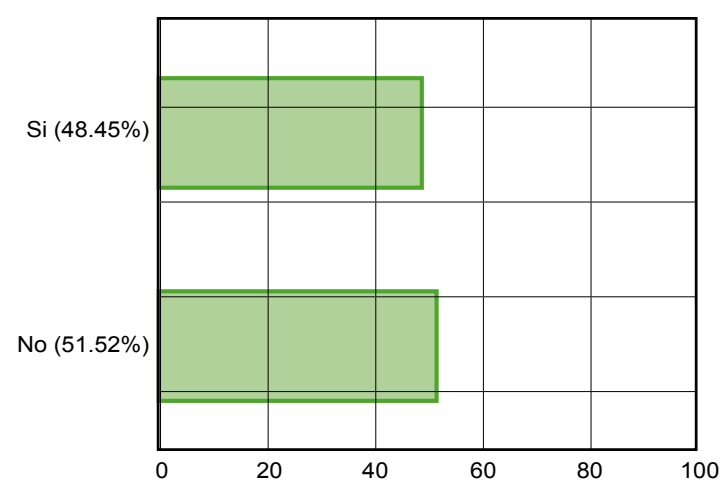

Figura No. 11. Acceso a internet en casa

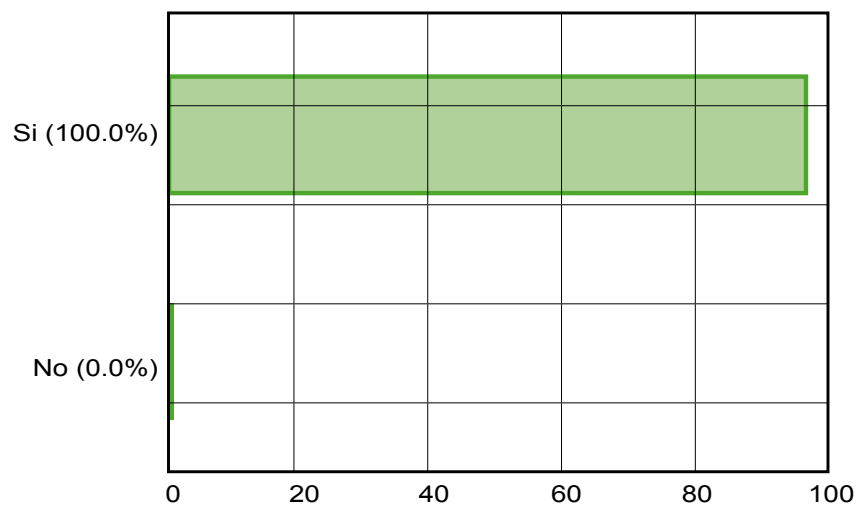

Figura No. 12. Utilidad de aplicación que le permita controlar su tratamiento

\section{RESULTADOS Y DISCUSIÓN}

Evaluando los hallazgos mediante la encuesta da una nueva perspectiva del impacto y los alcances. El porcentaje de los rangos de edades no permite decir que la incidencia se da entre 40 a 60 años, por ellos es necesario crear interfaces intuitivas, fáciles manejar, no sobre cargas para la aceptación de la plataforma por parte de la población. 


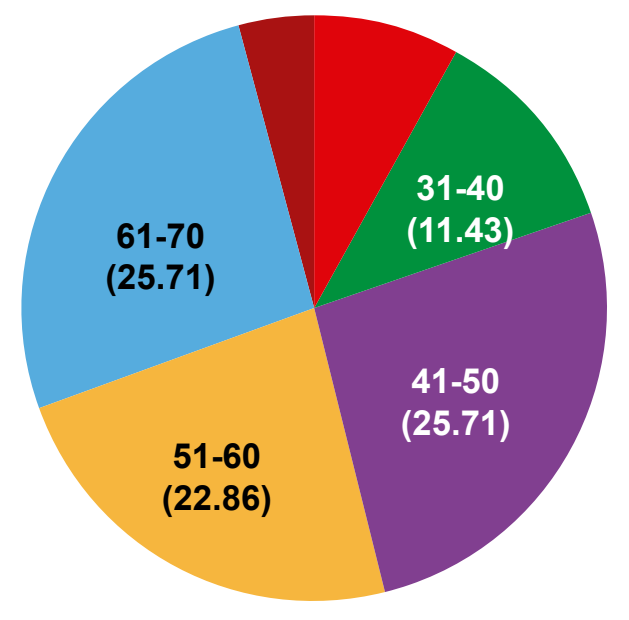

Figura No. 13. Rangos de Edad en Pacientes

Agregado a esto por la edad de paciente puede sufrir de problema de salud visual para lo que debe tomarse en cuenta el tamaño de los elementos en la interfaces, asimismo el tamaño de la fuente y el estilo que se desea usar. Al momento de consultarle a los pacientes por la reprogramación de las citas, los pacientes respondieron un como mayor que nunca se dio el caso. Este resultado puede ser por temor de contestar lo contrario.

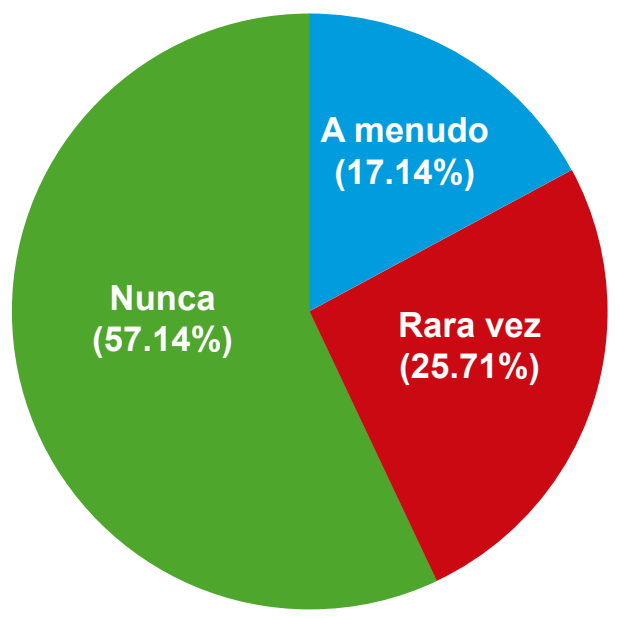

Figura No. 14. Frecuencia de Reprogramaciones de citas

A pesar de estos resultados la plataforma proveería al centro médico, porcentajes más bajos sobre el cambio de citas, reconociendo la frecuencia de reprogramación de las citas se debe tomar en cuenta por qué razón hace el cambio. 


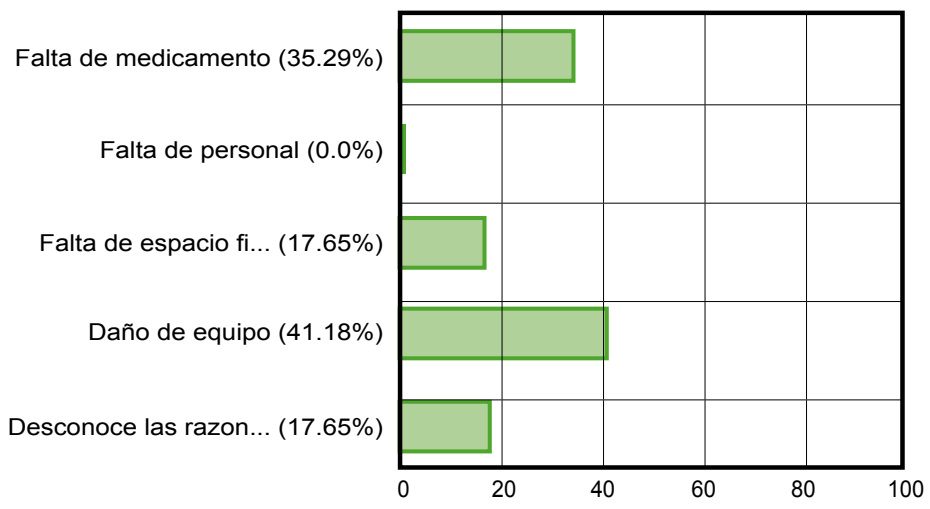

Figura No. 15. Frecuencia de Reprogramaciones de citas

Para establecer una plataforma principalmente para dispositivos móviles debe evaluarse en uso que hacen los pacientes o sus familiares, determinando la factibilidad del sistema de notificaciones.

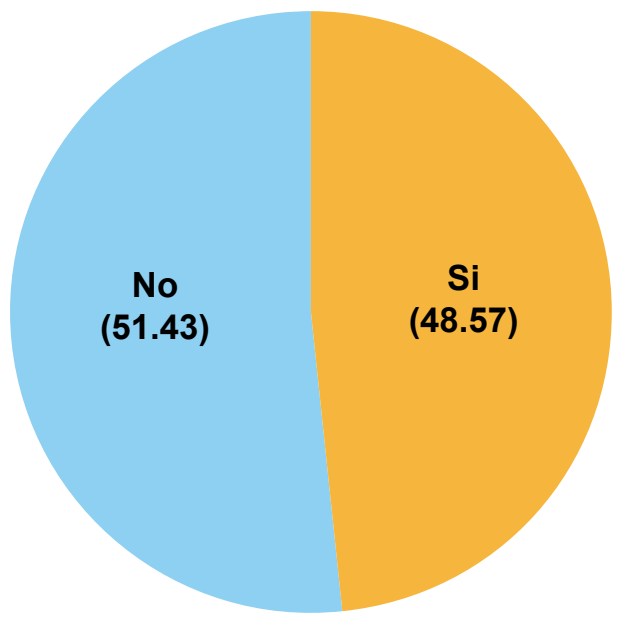

Figura No. 16. Uso de Dispositivos Inteligentes

En los hallazgos se logra conocer la plataforma o sistema operativo que usan los pacientes con mayor frecuente en dispositivos inteligentes, permitiendo establecer el mercado que tendrá la plataforma de notificaciones. 


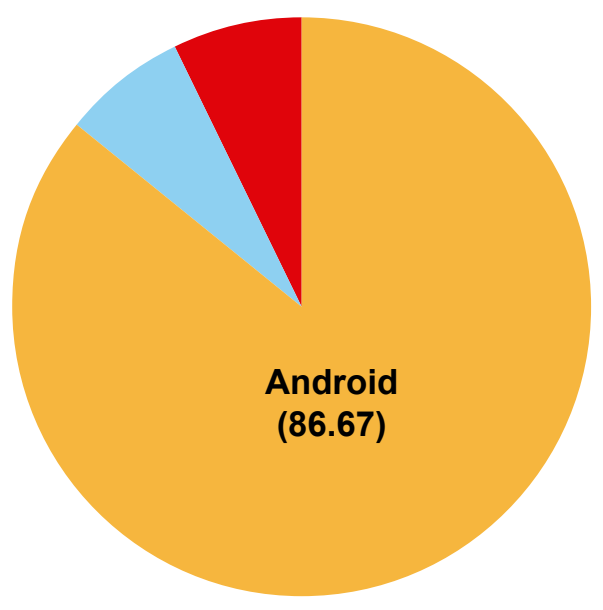

Figura No. 17. Sistema Operativo más usado

El uso de la plataforma debe estar enmarcado en el tipo de servicio de internet o acceso a datos con el que cuentan los pacientes, actualmente los proveedores de estos servicios ofrecen una diversa ofertas y promociones para las necesidades, beneficiando el uso de esta plataforma.

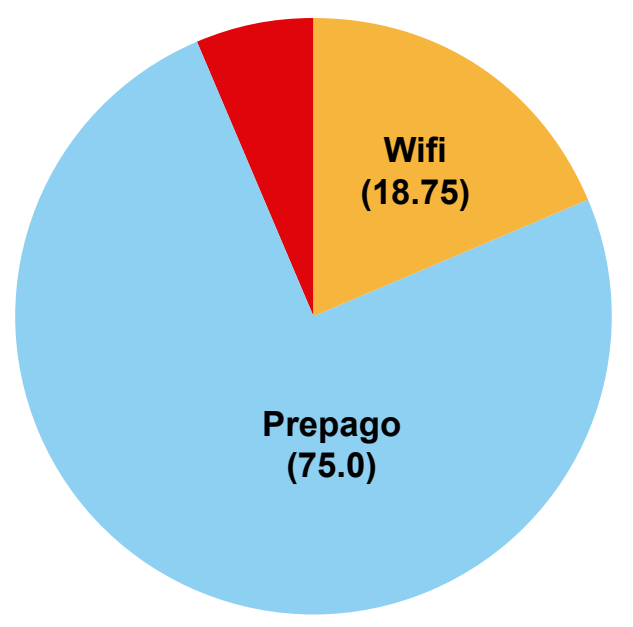

Figura No. 18. Conexión de Datos Móvil 


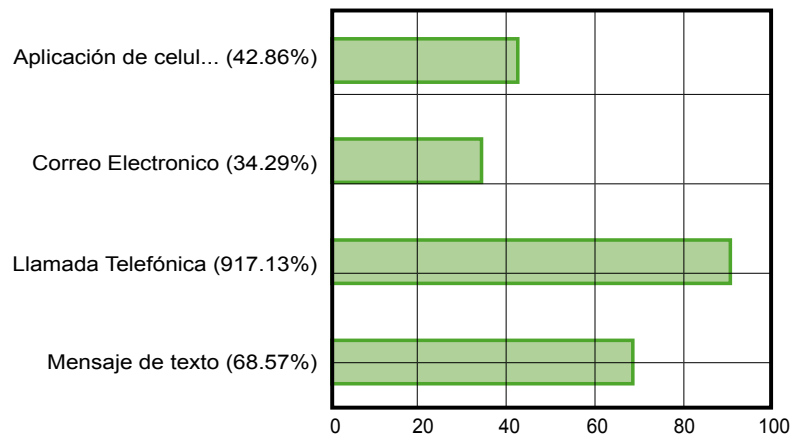

Figura No. 19. Medios para Notificaciones

\section{CONCLUSIONES E IMPLICACIONES}

El uso de tecnología en diversas áreas es una realidad, por lo que vemos que se vuelve parte vital en el desarrollo de las ciudades, país y regiones, a pesar de esto no buscar promover el desarrollo tecnológico, en otros casos es realmente escaso. En esta investigación un punto clave era reconocer el impacto que tendría el desarrollo tecnológico, dando como resultado el $100 \%$ de los encuestados que desean tener una plataforma de notificaciones.

En áreas sensitivas con Oncología debe llevarse un seguimiento del tratamiento para la mejora del paciente de igual manera ellos requiere que se les notifique e informe sobre su tratamiento, medicamentos y sus efectos, dinásticos e información de su enfermedad.

La investigación provee del medio por que los pacientes prefieren recibir las notificaciones y la información que les provea la Sala de Oncología. 


\section{REFERENCIAS BIBLIOGRÁFICAS}

Tratamiento contra el cáncer. (2015). Retrieved from http://www.cancer.net/es/desplazarse-por-atenci\%C3\%B3n-del-c\%C3\%A 1ncer/el-c\%C3\%A 1ncer-en-adultos-mayores/tratamiento-contra-el-c\%C3\%A1ncer

¿Qué es la eSalud? Definición y concepto. Retrieved from http://laesalud.com/que-esesalud/

"El 70 por ciento de la atención hospitalaria se podría llevar al hogar". Retrieved from http://www.aunclicdelastic.com/el-70-por-ciento-de-la-atencion-hospitalaria-se-podria-llevar-al-hogar-2/

Historia del Hospital San Felipe. Retrieved from http://www.salud.gob.hn/documentos/ hospitales/hitoria\%20hospital\%20san\%20felipe.pdf

Importancia de las TIC (tecnologías de la información y la comunicación) en el cuidado dela salud. Retrieved from http://www.clinicamedellin.com/importancia-de-las-tic-tecnologias-de-la-informacion-y-la-comunicacion-en-el-cuidado-de-la-salud/\#.VcMd-_l_Oko

Tecnología móvil para la salud. Retrieved from http://fira-news.com/es/tecnologia-movil-para-la-salud/

Tecnologías móviles al servicio de la salud. Retrieved from http://www.elfinanciero. com.mx/opinion/tecnologias-moviles-al-servicio-de-la-salud.html

Digital, C. C. Las TIC en los retos del sector salud. Retrieved from http://www.colombiadigital.net/herramientas/nuestras-publicaciones/sociedad-y-calidad-de-vida/ item/5261-las-tic-en-los-retos-del-sector-salud.html

Organización Mundial de la Salud. OMS | Cáncer. Retrieved from http://www.who.int/ mediacentre/factsheets/fs297/es/

World Health Organization. OMS | Cáncer. Retrieved from http://www.who.int/mediacentre/factsheets/fs297/es/

World Health Organization. OMS | La batalla mundial contra el cáncer no se ganará únicamente con tratamiento. Retrieved from http://www.who.int/mediacentre/news/ releases/2014/cancer-report-20140203/es/ 